\title{
Recovery of probiotic bacteria from the intestinal tract of broilers after noninvasive pre-hatch application
}

\author{
I. Thøfner ${ }^{1}$, D. Sandvang ${ }^{2}$, K. Aagaard ${ }^{1}$ and L. Ladefoged Poulsen ${ }^{1 *}$ \\ ${ }^{1}$ University of Copenhagen, Department of Veterinary and Animal Sciences, Faculty of Health and Medical Sciences, \\ Stigboejlen 4, 1870 Frederiksberg, Denmark; ${ }^{2}$ Chr. Hansen A/S, Animal Health Innovation, Boege allé 10, 2970 Hoersholm, \\ Denmark; ladefog@sund.ku.dk
}

Received: 15 December 2020 / Accepted: 14 June 2021

(c) 2021 Wageningen Academic Publishers

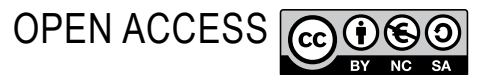

RESEARCH ARTICLE

\begin{abstract}
This paper reports the success of intestinal colonisation of chickens and foetuses by probiotics after different methods of pre-hatch application. Hatcheries not using in ovo injection of probiotics or wish to avoid the reduced hatchability associated with in ovo injections prefer using alternatives to in ovo technologies. Therefore, we used noninvasive pre-hatch application methods. This included the vertical transmission of probiotics from the mother hen to offspring, application of probiotic late in incubation and transmission of probiotics during hatch. Enterococcus faecium (NCIMB11181) and Lactobacillus animalis (DSM33570) were used as probiotics. Probiotics were applied either through drinking water for the mother hens, by dipping the eggs in a probiotic solution on days 16-18 of incubation or through drops/spray on the eggshell of the fertilised eggs. Similarly, intestinal colonisation of the probiotic in chickens was investigated either before hatch (pre-hatch) or immediately after hatch (post-hatch). Based on the performed experiments, it is concluded that $E$. faecium was vertically transmitted from the mother hen to the offspring, as E. faecium was recovered in 20 and $33 \%$ of the offspring pre- and post-hatch, respectively. When applied on the eggshell, the recovery of E. faecium before hatch depended on the application method and ranged from 0 to $9 \%$. In contrast, $L$. animalis was not recovered before hatch. Moreover, when sampling post-hatch $100 \%$ of the chickens were colonised when E. faecium was used and $54 \%$ were colonised when L. animalis was used. Furthermore, spray application with E. faecium was the most successful application method as $9 \%$ of the foetuses were colonised pre-hatch and 100\% became colonised post-hatch. Therefore, pre-hatch application by, for example, spray of probiotics on the eggshell can be used as an easy-to-use, noninvasive method for early life colonisation of chicken gut.
\end{abstract}

Keywords: probiotics, chickens, noninvasive application, intestinal colonisation, pre-hatch

\section{Introduction}

Probiotics for poultry have traditionally been added to feed or water. However, applying probiotics pre-hatch is proposed to have several advantages. Most importantly, the probiotic bacteria have better and longer-lasting effects when chickens are exposed to probiotic bacteria before intestinal microflora develops (Kubasova et al., 2019). Before the establishment of the intestinal flora, the competition for adhesion sites and nutrients in the intestine is lower than in a more developed gastrointestinal microflora as found in older chickens. This means that the probiotic bacteria have a better chance of colonising the intestine (Dominguez-Bello et al., 2011; Oakley et al., 2014; Patterson and Burkholder 2003; Salminen and Isolauri 2006; Stanley et al., 2014). Chickens colonised by beneficial probiotic bacteria at hatch are expected to be less susceptible to pathogenic bacteria during the critical first week of life. Furthermore, when chickens hatched in industrialised hatchers, they are not exposed to the bacterial flora from the mother hen but to bacteria originating from exploding rotten eggs, bacteria from chickens carrying a vertically transmitted infection from the mother hen and bacteria that are carried over from previous hatchings 
(Kubasova et al., 2019; Poulsen et al., 2017; Stanley et al., 2013). Therefore, applying probiotic bacteria pre-hatch is an initiative to reduce colonisation and spread of potentially pathogenic bacteria, which the newly hatched chickens are exposed to in the hatcher and on the farm. Additionally, to exert protective effects against bacterial infections, some probiotics (Lactobacillus spp.) have been demonstrated to have a positive effect on growth performance of broilers (Timmerman et al., 2006; Zulkifli et al., 2000).

Traditionally, pre-hatch administration of vaccines as well as probiotics and feed supplements (to a lesser extent) have been applied as in ovo injections. However, the success and safety (e.g. hatchability, and embryonic/foetal death) of this type of application highly relies on the injection site, hatchery practice and agent of interest (Peebles, 2018; Retes et al., 2018). To avoid a reduction in hatchability due to in ovo injections, alternative pre-hatch application methods are of interest.

Therefore, this study investigates the applicability of noninvasive pre-hatch application of probiotics for early life colonisation of the chicken gut. This study aimed to specifically investigate the following:

1. whether the transmission of probiotics is possible from the mother hen to the offspring by vertical transmission;

2. whether probiotic applied on the shell of eggs late during incubation can colonise the unhatched chicks;

3. whether horizontal transmission of probiotics occurs during hatch;

4. whether colonisation before and during hatch depends on the probiotic bacteria used; and

5. whether spray application with probiotic pre-hatch is equally as effective as in ovo injections with probiotics when measuring colonisation on day 7 post-hatch.

\section{Materials and methods}

\section{Application methods and sampling design}

Two strategies for the noninvasive application of probiotics to chicken before hatch were investigated. The first strategy was to apply the probiotic indirectly to the broilers by administering the probiotic (Enterococcus faecium) to the drinking water of the parent birds (broiler breeders) (Figure 1A). This process will be henceforth referred to as 'vertical transmission' in the manuscript. The second strategy was to apply the probiotic directly to the fertilised eggs between days 16 and 18 of incubation. This strategy was investigated using three application methods of the probiotic onto the egg surface: (1) dipping the eggs in a probiotic solution or (2) applying in drops or (3) spraying onto the surface (Figure 1B-D). Morever, the rate of intestinal colonisation by the probiotics before and after hatch was evaluated. Specific application methods for each strategy are described in detail in the sections below.

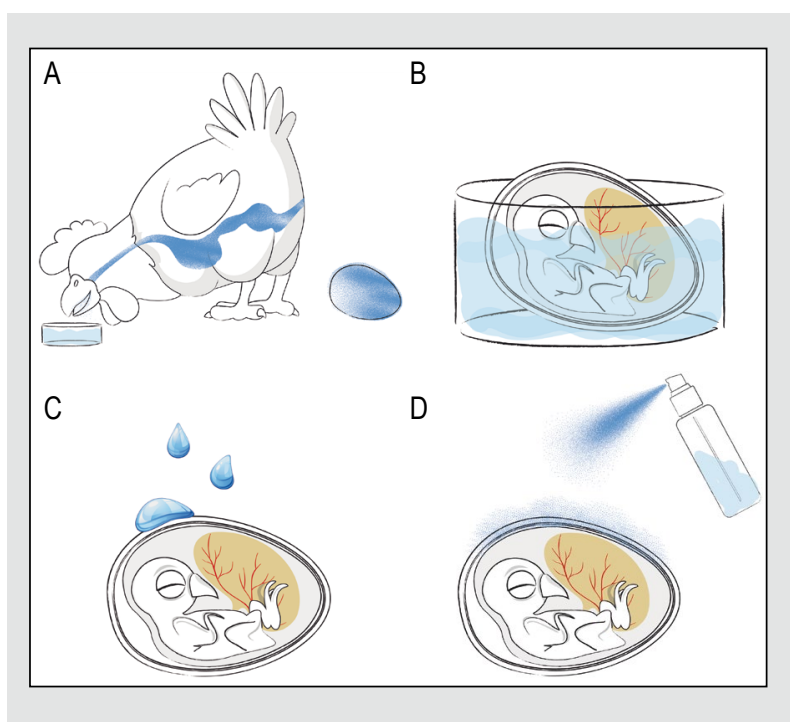

Figure 1. An illustration of the different application methods used in the experiments.

\section{Probiotic strains}

Lyophilised E. faecium (NCIMB 11181), $2 \times 10^{11} \mathrm{cfu} / \mathrm{g}$, and Lactobacillus animalis (DSM33570), $3 \times 10^{10} \mathrm{cfu} / \mathrm{g}$, were used for pre-hatch application on fertilised eggs. These strains are referred to as E. faecium and L. animalis, respectively, in the manuscript henceforth.

\section{Application of Enterococcus faecium in drinking water for broiler breeders (vertical transmission) (Objective 1)}

To investigate the possible vertical transmission of probiotics from the mother hen to offspring, E. faecium was suspended daily in drinking water for 10 broiler breeders aged 44 weeks at the onset of the trial. They had unlimited access to water, with an estimated daily water consumption of 262-328 ml water/hen/day (Aviagen, 2016, 2018). To obtain a final concentration of approximately $1 \times 10^{8} \mathrm{cfu} / \mathrm{ml}$, resulting in an estimated average daily dose of $2.6-3.3 \times 10^{10}$ cfu/hen, $0.5 \mathrm{~g}$ of lyophilised E. faecium (NCIMB 11181) was suspended per litre tap water. The probiotic administration through drinking water to broiler breeders continued until egg collection was discontinued at the end of the trial. Eggs collected before the application of probiotics were used as negative control eggs. All eggs were stored separately at $17^{\circ} \mathrm{C}$ until incubation.

\section{Housing of broiler breeders and collection of eggs}

Ten broiler breeder hens (Ross 308), 44 weeks old, and two roosters (Ross 307 ) were evenly distributed in two pens (approximately $8 \mathrm{~m}^{2} /$ pen). Perches, nests and sand for dust bath were provided in each pen. Feed was restricted to $164 \mathrm{~g}$ per hen or rooster and water was applied ad libitum. 
Eggs were laid in nests and collected daily. The eggs were collected using sterile gloves and stored in sterile egg trays without physical contact with other eggs to prevent crosscontamination between eggs.

\section{Application of Enterococcus faecium and Lactobacillus animalis on the eggshell surface (Objectives 2-4)}

The colonisation potential of probiotics applied on the eggshell of fertilised eggs were investigated in the unhatched and newly hatched chickens. For investigating the colonisation of unhatched chickens, sampling of intestine and yolk sac occurred before hatching (incubation day 19) as described in Tables 1-4, whereas for the investigation of colonisation during/after hatching, the sampling occurred during or immediately after hatching (incubation days 20-21) (Tables 1-4). Horizontal transmission of probiotic bacteria was described in experiments in which more chickens were colonised right after hatch than before hatch.

For the dipping experiment (Figure 1B), eggs were soaked for $1 \mathrm{~s}$, left to dry for $1 \mathrm{~min}$ and soaked again for another second in an E. faecium solution $\left(7 \times 10^{10} \mathrm{cfu} / \mathrm{ml}\right)$ (Table S1, Experiments 3 and 4). When applying E. faecium as a drop on the eggshell (Figure $1 \mathrm{C}$ and Table 2), $50 \mu \mathrm{l}$ was pipetted onto the eggshell on the side or at the blunt end of the egg after moving to the hatching trays. When the spray method was used to apply the probiotic, the amount of probiotic solution distributed per pump of the spray flask was calculated by weighing 10 pumps. Subsequently, the average of this value for calculating volumes was used with the aim of applying approximately $50 \mu \mathrm{l} / \mathrm{egg}$ (Figure 1D; Tables 3 and 4). For each experiment, the $\mathrm{cfu} / \mathrm{ml}$ was determined, and the volume used per egg was then used for calculating the cfu used for each egg in the experiments.

For eggshell applications, the sham-inoculation method with sterile isotonic saline by dip, drop or spray was used on control eggs as described above according to the application method in use. For every eggshell application, care was taken to minimise cooling stress on the eggs during the application procedures.

\section{In ovo application of Enterococcus faecium (Objective 5)}

To investigate if spray application was equally effective as in ovo injection of the probiotic, a comparison of the cfu in the caecum on day 7 was performed on chickens that

Table 1. Application of Enterococcus faecium in drinking water for broiler breeders (Objective 1).

\begin{tabular}{lllllll} 
Hatch status & Day of application & Day of sampling & Probiotic & $\mathbf{n}$ & cfu/eg $^{\mathbf{1}}$ & Recovery rate of probiotic, $\mathbf{n}(\%)$ \\
Unhatched & daily & 19 & E. faecium & 20 & $3 \times 10^{10}$ cfu/hen/day & $4(20)$ \\
& & & saline & 20 & $-^{2}$ & 0 \\
Hatched & \multirow{2}{*}{21} & E. faecium & 33 & $3 \times 10^{10}$ cfu/hen/day & $11(33)$ \\
& & saline & 20 & $-^{2}$ & $11(55)$ \\
\hline
\end{tabular}

${ }^{1}$ Exact dose of application cannot be calculated because it relied on the water intake of the hen.

${ }^{2}$ Not applicable as sampling occurred pre-hatch.

Table 2. Application of Enterococcus faecium as a drop on the eggshell (Objectives 2-4).

\begin{tabular}{|c|c|c|c|c|c|c|c|}
\hline Application site & Hatch status & $\begin{array}{l}\text { Day of } \\
\text { application }\end{array}$ & $\begin{array}{l}\text { Day of } \\
\text { sampling }\end{array}$ & Probiotic & $\mathrm{n}$ & cfu/egg & $\begin{array}{l}\text { Recovery rate of } \\
\text { probiotic, } n(\%)\end{array}$ \\
\hline \multirow[t]{4}{*}{ Drop at side of egg } & unhatched & 17 & 19 & E. faecium & 46 & $6 \times 10^{8}$ & $3(7)$ \\
\hline & & & & saline & 40 & - & 0 \\
\hline & hatched & 17 & 20 & E. faecium & $30^{\mathrm{a}}$ & $3 \times 10^{8}$ & $25(83)$ \\
\hline & & & & saline & 40 & - & 0 \\
\hline \multirow[t]{4}{*}{ Drop at blunt end of egg } & unhatched & 17 & 20 & E. faecium & $32^{b}$ & $3 \times 10^{8}$ & $0(0)$ \\
\hline & & & & saline & 40 & - & 0 \\
\hline & hatched & 17 & 20 & E. faecium & $14^{\mathrm{C}}$ & $3 \times 10^{8}$ & $6(43)$ \\
\hline & & & & saline & 40 & - & 0 \\
\hline
\end{tabular}

\footnotetext{
a Sampling occurred on day 20 . Only fully hatched chickens were included.

b Sampling occurred on day 20. Hatched chickens were excluded.

c Sampling occurred on day 20. Only fully hatched chickens were included.
} 
Table 3. Application of Enterococcus faecium as spray on the eggshell (Objectives 2-4).

\begin{tabular}{|c|c|c|c|c|c|c|c|}
\hline Hatch status & Day of application & Day of sampling & Probiotic & $\mathrm{n}$ & cfu/egg & $\begin{array}{l}\text { Recovery rate of } \\
\text { probiotic, } n(\%)\end{array}$ & Hatchability (\%) \\
\hline \multirow[t]{2}{*}{ Unhatched } & 17 & 19 & E. faecium & 47 & $6 \times 10^{8}$ & $4(9)$ & - \\
\hline & & & saline & 40 & - & 0 & - \\
\hline \multirow[t]{2}{*}{ Hatched } & 16 & 21 & E. faecium & 46 & $4 \times 10^{8}$ & $46(100)$ & 94 \\
\hline & & & saline & 40 & - & 0 & 93 \\
\hline
\end{tabular}

Table 4. Application of Lactobacillus animalis as spray on the eggshell (Objectives 2-4).

\begin{tabular}{|c|c|c|c|c|c|c|c|}
\hline Hatch status & Day of application & Day of sampling & Probiotic & $\mathrm{n}$ & cfu/egg & $\begin{array}{l}\text { Recovery rate of } \\
\text { probiotic, } n(\%)\end{array}$ & Hatchability (\%) \\
\hline \multirow[t]{2}{*}{ Unhatched } & 18 & 19 & L. animalis & 33 & $1 \times 10^{7}$ & 0 & - \\
\hline & & & saline & 37 & - & 0 & - \\
\hline \multirow[t]{2}{*}{ Hatched } & & 21 & L. animalis & 33 & $1 \times 10^{7}$ & $18(54)$ & 83 \\
\hline & & & saline & 29 & - & 0 & 85 \\
\hline
\end{tabular}

received the probiotic on day 18 in the incubation period. The in ovo experiment was conducted at the Mississippi State University, where E. faecium was administered in ovo with Marek's vaccine (Zoetis, Parsippany, NJ, USA) in the amnion cavity (Skjøt-Rasmussen et al., 2019). A selection of these samples was analysed in this study and compared with samples from the spray application (Table 5, Supplementary Table S1 - Experiment 18).

\section{Enumeration of probiotic solutions administered}

Serial 10-fold dilutions were used in the enumeration of viable bacterial counts (cfu) in all probiotic solutions. Subsequently, plating of $100 \mu \mathrm{l}$ of each dilution on a blood agar (BA) plate prepared with $5 \%$ calf blood on BA base (Oxoid, Basingstoke, UK) for E. faecium and De Man, Rogosa and Sharpe's (MRS's) broth (Oxoid) for L. animalis was conducted. Next, agar plates were incubated aerobically (E. faecium) and anaerobically (L. animalis) at $37^{\circ} \mathrm{C}$ overnight before counting. The cfu platings were conducted three times.

\section{Fertilised eggs}

For the probiotic application on the eggshell, preincubated fertilised eggs (Ross 308, incubation days 0-16) were obtained from a commercial hatchery in Denmark. Additionally, fertilised Layer-type eggs (LSL-Lite) from another Danish commercial hatchery were used.

\section{Incubation and hatching}

Incubation and hatching of eggs was conducted in Heka hatchers for 160 or 360 eggs (America A/S, Thisted, Denmark). Separate hatchers were used for the control and the probiotic-treated eggs. Incubation temperature was set at $37.7^{\circ} \mathrm{C}$. The humidity was set at approximately $65 \%$, and automatic turning were conducted until 4 days before hatch, when the eggs were placed into hatching trays. Unfertilised eggs were removed from the hatchers after candling between day 10 and 14 .

Table 5. Application of Enterococcus faecium as in ovo injection (Objective 5).

\begin{tabular}{|c|c|c|c|c|c|c|c|}
\hline Hatch status & Day of application & Day of sampling & Probiotic & $\mathrm{n}$ & cfulegg & $\begin{array}{l}\text { Recovery rate of } \\
\text { probiotic, } n(\%)\end{array}$ & Hatchability (\%) \\
\hline Hatched & 18 & 7 post-hatch & $\begin{array}{l}\text { E. faecium with } \\
\text { Marek's vaccine }\end{array}$ & $10^{\mathrm{a}}$ & $1 \times 10^{7}$ & 100 & 81 \\
\hline
\end{tabular}

a Ten samples were analysed in this study, and the remaining samples were analysed by Skjøt-Rasmussen et al. (2019). 


\section{Post-application bacteriology of unhatched and newly hatched chickens (Objectives 1-5)}

Intestinal colonisation by probiotics was investigated at different time points in relation to hatching (Tables 1-5). Decapitation was then performed to euthanise newly hatched chickens and unhatched foetuses. To reduce the risk of contamination during sampling of the internal organs, every egg, euthanised foetus and chicken was dipped in $70 \%$ ethanol and allowed to dry for few minutes before sampling. Entire intestine and yolk sac were aseptically removed with sterile scissors and tweezers, after which the equipment and gloves were changed for every chicken to prevent cross-contamination. Yolk sac and intestines were used for re-isolation of E. faecium, both pre-hatch and immediately post-hatch. Subsequently, samples (yolk sac/ intestine) were homogenised with sterile isotonic saline (1:1 ratio) until homogeneity. Next, the samples were pre-incubated aerobically at $37^{\circ} \mathrm{C}$ in $30 \mathrm{ml}$ of brain heart infusion (BHI) broth (Oxoid) when E. faecium was used as a probiotic. In contrast, when $L$. animalis was used as a probiotic, samples were incubated in MRS's broth (Oxoid) and incubated anaerobically.

After overnight incubation, a $10 \mu \mathrm{l}$ inoculation needle was used to streak the enriched BHI broth on BA containing kanamycin (BA-kan) $(1000 \mu \mathrm{g} / \mathrm{ml})$ (Sigma-Aldrich, Soeborg, Denmark) and incubated overnight. In the experiment where L. animalis was re-isolated, MRS broth was used for pre-incubation, after which samples were streaked on MRS containing kanamycin and vancomycin (200 and $300 \mu \mathrm{g} / \mathrm{ml}$, respectively). Further, sub-culturing from the primary BA/MRS was performed in cases in which the yolk sac/intestinal material on the agar plate masked the analysis. In the experiment where L. animalis was applied five times, the yolk sac/intestinal samples were spiked with $1 \times 10^{7} \mathrm{cfu}$ as positive controls.

\section{Verification of the probiotic Enterococcus faecium and Lactobacillus animalis by strain-specific PCR}

DNA was extracted following the boiling lysate method. Here, one loop (size of loop $1 \mu \mathrm{l}$ ) of the bacterial colony was suspended in $300 \mu \mathrm{l}$ of Milli-Q water (vortex) and heated for $10 \mathrm{~min}$ at $100{ }^{\circ} \mathrm{C}$. Then, the setup was centrifuged for $5 \mathrm{~min}$ at $14,000 \times g$ before the supernatant was transferred to a new tube and used as the DNA template.

Alternatively, DNA was extracted with the Maxwell RSC Instrument (Promega, Lystrup, Denmark) using the Maxwell RSC Cultured Cells DNA Kit (AS 1620) (Promega). Before DNA extraction, a single colony from the samples was sub-cultivated on BA (without kanamycin) to obtain a pure culture. The PCR programmes are described in the supplementary material. Strain-specific primers were developed and used for detecting probiotic bacteria by PCR (Table 6).
Table 6. Strain-specific primers used to detect Enterococcus faecium and Lactobacillus animalis.

$\begin{array}{lll}\text { Primer name } & \text { Sequence }\left(\mathbf{5}^{\prime} \rightarrow \mathbf{3}^{\prime}\right) & \begin{array}{l}\text { Amplicon } \\ \text { size (bp) }\end{array} \\ \text { E. faecium_F5 } & \text { AGAACAGAGAAGTAGACCAGCCA } & 920 \\ \text { E. faecium_R5 } & \text { TGAGGCTGCGATGTTGAAGT } & \\ \text { L. animalis_F } & \text { AGCAACTCTTCCCAGATGAACA } & 114 \\ \text { L. animalis_R } & \text { AGCCGGAAATATAGTTAGTCGCA } & \end{array}$

Moreover, $1 \%$ agarose gels were used to separate amplicons by electrophoresis (125 V in $40 \mathrm{~min}$ ). Then, Generuler 1 kb plus DNA ladder (Catalogue Number SM1331; Thermo Scientific, Waltham, MA, USA) was used as a marker; bands were visualised with a Bio-Rad Molecular Imager ChemiDoc $^{\text {TM }}$ XRS + (Bio-Rad, Copenhagen, Denmark) using the Image Lab ${ }^{\text {ts }}$ Software (6.0 Bio-Rad).

\section{Verification of the specific probiotic Enterococcus faecium strain by pulsed-field gel electrophoresis}

From the experiments investigating vertical transmission of the probiotic strain from the hen to offspring, 10 random isolates were selected for verification by pulsed field gel electrophoresis. The analysis was performed as described by Jørgensen et al. (2017).

\section{Investigating the presence of Enterococcus faecium in the salpinx of probiotic-treated broiler breeder hens}

After the application of probiotic in drinking water, the egg-laying hens were euthanised. Salpinx was then removed from hens $(n=8)$, and the mucus layer of the proximal half of the magnum and the distal part of the isthmus were scraped with a glass slide. Next, the mucus from each sample was streaked with a sterile cotton swab on BA-kan plates. Growth was described semi-quantitatively $(0=$ no growth, 1 = colonies in the primary streak, 2 = colonies in the second streak and $3=$ colonies all the way to the third streak). Verification of the probiotic strain was conducted using the strain-specific PCR described above.

\section{Re-isolation of the probiotic Enterococcus faecium from caeca of the chickens' day 4 and 7 post-hatch after application by spray and in ovo injection (Objective 5)}

To compare how the application method affected colonisation of the chicken intestinal tract (caeca) on day 7 post-hatch E. faecium was applied as a spray and by $i n$ ovo injection. At first, 120 eggs were sprayed on day 18 of incubation with $2 \times 10^{8} \mathrm{cfu} / \mathrm{egg}$. The newly hatched chickens $(n=120)$ were housed in an animal facility (floor pens with wood shavings, perches and feed and water ad libitum) 
until day 7. The control group and the probiotic treated group were housed in separate rooms to prevent crosscontamination (Supplementary Table S1 - Experiment 14). Chickens were weighed on days 1, 4 and 7. Subsequently, the presence of E. faecium was investigated at two time points (days 4 and 7) by euthanasia of 30 chickens from each group. Sampling was performed as described in the section 'Post-application bacteriology of unhatched and newly hatched chickens'. Apart from that, only the caeca were used instead of the yolk sac/intestine for enumeration of the E. faecium probiotics. Caecal samples were stored at $-20{ }^{\circ} \mathrm{C}$ until analysis. One caecum per chicken was homogenised with saline (1:1 ratio). The samples were 10 -fold diluted, after which $2 \times 10 \mu \mathrm{l}$ was spotted on BA-kan and incubated aerobically overnight. The following day, cfu was determined. From each sample, one colony was subcultivated on a BA-kan and used for strain verification by PCR as described above.

The efficacy of the spray application was compared with that of in ovo application. Enumeration was used for this comparison of the E. faecium in caeca of 10 chickens (Ross 708 ), which received the E. faecium probiotic via in ovo injection. These chickens received a concentration of $1.4 \times 10^{7}$ cfu E. faecium/egg into the amniotic fluid as previously described by Skjøt-Rasmussen et al. (2019). Their caeca were stored separately in cryotubes at $-80^{\circ} \mathrm{C}$ until analysis was conducted, together with the caecal samples from the spray experiment described above.

\section{Ethics}

The animal experiments were conducted in agreement with the Danish animal expectorate under the licence number 2019-15-0201-01619.

\section{Statistics}

The results are presented as rates (e.g. hatchability and reisolation of probiotic) and means with standard deviation (SD) (cfu and body weight). For normalising cfu counts, $\log 10$ transformation was applied. All statistical analysis was performed using Prism 8 for Windows (version 8.4.0, GraphPad Software, Inc., La Jolla, CA, USA). The Chisquare test was used for comparing colonisation rates of the pre- and post-hatch intestines; Fisher's exact test was used to compare differences in hatchability. In addition, one way ANOVA followed by Tukey's multiple comparison test was used to calculate differences in the body weight on days 1, 4 and 7 and for comparing cfu values on days 4 and 7 after application by spray and in ovo injection, respectively. Significance levels were set to 0.05 .

\section{Results}

The results from different application experiments are summarised in Tables 1-5. Results concerning the specific study objectives 1-5 are described in detail in the sections below.

\section{Vertical transmission of $E$. faecium from breeders to broilers and the presence of $E$. faecium in the salpinx of the probiotic-treated breeders}

The probiotic E. faecium passed from the mother hen to the offspring (Table 1). In the unhatched chickens, the probiotic strain was recovered from $20 \%$ of the chicks at the time of hatch, whereas $33 \%$ of the chicks had the probiotic strain in their intestine/yolk sac. These results are illustrated as the first bars in Figure 2 ('Vertical'). Although the incubation of eggs occurred in different hatchers, $55 \%$ of the untreated control chickens were colonised at hatch. The two hatchers were then placed in the same room, after which the hatcher used for the control eggs was subsequently found to be contaminated with the probiotic strain. After sampling, an open petri dish with BHI was placed in the hatcher for $4 \mathrm{~h}$. Next, $100 \mu \mathrm{l}$ of the broth was spread on a BA-kan plate and incubated overnight at $37^{\circ} \mathrm{C}$. Furthermore, it was discovered that the ventilation outlet in the hatcher was unfiltered. Therefore, control chickens were colonised during hatch in the control hatcher. Subsequently, the hatchers were disinfected and the control hatcher was placed in a separate room for future experiments.

From the investigation of the broiler breeder hens, the probiotic strain was found to be present in the proximal part of the magnum of the salpinx in four of the eight hens by direct plating. The growth varied from limited (score 1 ) in one hen, moderate growth (score 2) in two hens and extensive growth (score 3 ) in one hen. In three of these four hens, the probiotic strain was found in the distal part of isthmus, one with limited growth (score 1) and two with extensive growth (score 3). In total, four of the eight had a salpinx that was positive for growth at one sampling site $(n=1)$ or two sampling sites $(n=3)$. Strain identity was confirmed by strain-specific PCR.

\section{Investigating colonisation of chickens pre-hatch}

When using E. faecium and sampling pre-hatch, none of the chickens were colonised after applying a drop at the blunt end (0/32). However, when applying the drop at the side of the eggs, 7\% (3/46) were colonised; when applying the probiotic as a spray on the side of the eggs, 9\% (4/47) of the yolk sacs/intestines became colonised. The highest pre-hatch colonisation rate was noted when the probiotic was applied in the drinking water for the mother hen in which $20 \%$ (4/20) of the offspring were colonised pre-hatch (Table 1). Moreover, in the experiment in which the eggs 
A

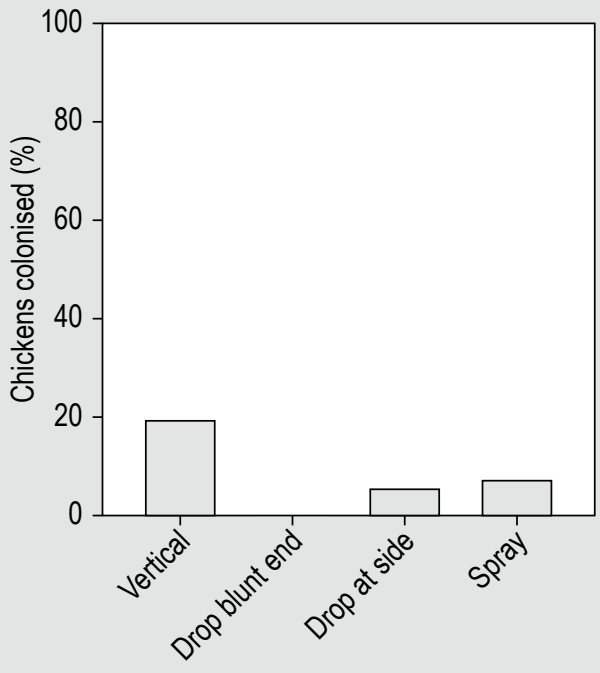

B

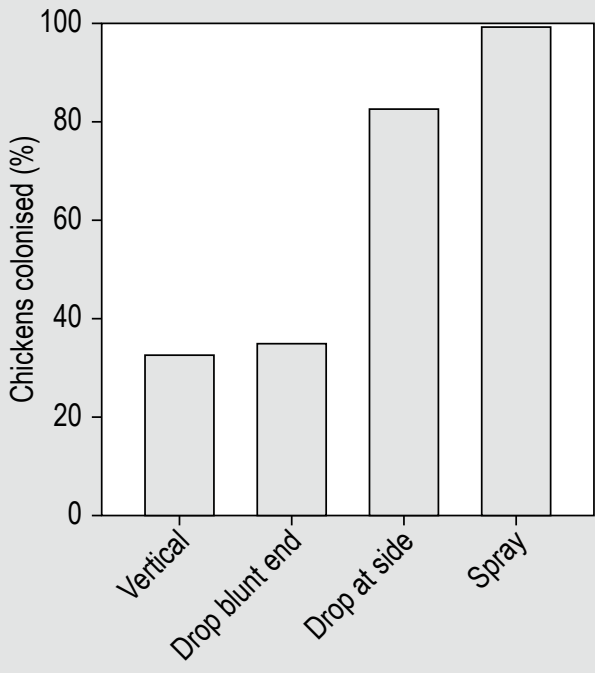

Figure 2. Percentage of chickens colonised pre-hatch (A) and post-hatch (B) with different application methods using Enterococcus faecium as probiotic.

were dipped twice in a concentration of $7 \times 10^{10} \mathrm{cfu} / \mathrm{ml}$, the re-isolation rate was $57 \%$ pre-hatch. At hatch, $100 \%$ were colonised; however, only $30 \%$ of the dipped chickens hatched (Supplementary Table S1 - Experiment 3 and 4).

The anatomy of the embryonated egg is illustrated in Figure 1. As shown, no blood vessels are present at the blunt end where the air cell is found. At the side of the egg, there is a higher probability that the probiotic bacteria came in close contact with blood vessels in either the vitelline or allantoic membranes, which facilitated the colonisation of the foetal intestine.

In contrast, when the probiotic was administered as a spray at the side of the eggs, the probiotic recovery was $9 \%$ in the unhatched foetuses. However, the L. animalis, which was applied as a spray (Table 3), was not re-isolated from any unhatched foetuses $(0 / 33)$.

\section{Investigating colonisation of chickens post-hatch}

When sampling chickens immediately after hatch, the rate of re-isolation of the probiotic strain varied according to the application method (Figure 2B). However, with vertical application of the probiotic to mother hen, $33 \%$ of the newly hatched chickens were colonised (Table 1, Figure 2B).

In the experiments in which a drop was placed at the side or the blunt end of the egg (Table 2), the re-isolation rates were 83 and $43 \%$, respectively. Similarly, in the experiments were spray was used as an application method, the re-isolation was $100 \%$ when treated with E. faecium (Table 3) and 54\% in the group treated with $L$. animalis (Table 4 ).

\section{Investigating caecal colonisation of 4- and 7-day old chickens after spray application and in ovo injection on day 18 of incubation}

The probiotic E. faecium strain was re-isolated from the caecum on days 4 and 7 concentrations stated in Figure 3. Control group chickens on days $4(n=30)$ and $7(n=30)$ were negative for the probiotic E. faecium. However, in the chickens originating from the sprayed eggs, the mean log (cfu) of the probiotic strain was significantly lower in the caeca of chickens on day 4 (mean $\log (\mathrm{cfu}) \pm$ SD: $6.27 \pm 1.37$ ) than on day $7(P=0.0007)$. Therefore, the chickens, which received the probiotic as an injection into the amniotic fluid, had a mean $\log$ (cfu) of $6.05 \pm 0.66$.

\section{Hatchability}

The hatchability did not differ after spray application on Ross 308 eggs with sterile media (placebo) or E. faecium. The hatchability varied from 93 to $94 \%$ (Table 3$)(P>0.9999)$. Likewise, no difference was observed on the hatchability after application of L. animalis in LSL-Lite eggs $(P=0.7379)$, resulting in hatchabilities on 83 and $85 \%$ in the treated and control groups, respectively (Table 4). However, in the in ovo injection trial, the control group was injected with Marek's vaccine alone, and hatchability where compared with that of the group injected with Marek's vaccine and the E. faecium probiotic. Based on the results, no significant difference in hatchability was found between these groups (Skjøt-Rasmussen et al., 2019). Only in the experiment in which eggs were dipped in the probiotic solution did the hatchability differ significantly between the control and treated groups (Supplementary Table S1 - Experiments 3 and 4). 


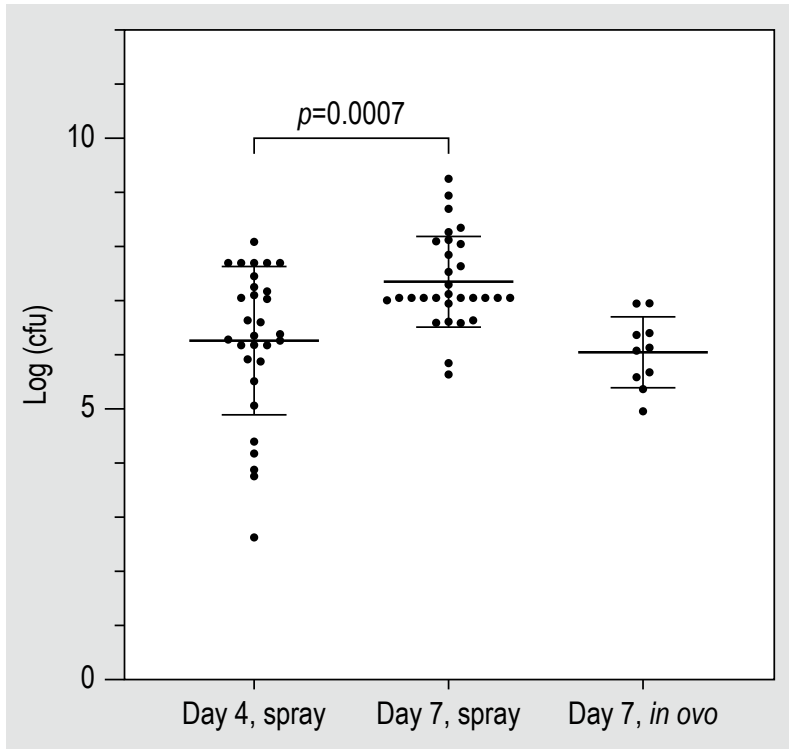

Figure 3. The mean log (cfu) of Enterococcus faecium per gram caeca in chickens on days 4 and 7 after spray application on the eggshell and in ovo injection (only day 7), with E. faecium probiotic on day 18 of incubation (Mean and standard deviation are shown).

The hatchability was $30 \%$ in the probiotic group, in which eggs were dipped in $7 \times 10^{10} \mathrm{cfu} / \mathrm{ml}$ E. faecium compared with that of the control group, in which eggs were dipped in saline. Furthermore, the unhatched chickens in the probiotic group (dip application) were found to be dead late in the incubation period. All dead chickens were investigated for pathogens as E. coli or E. faecalis in the yolk sac of the chickens. Similarly, hatchability after in ovo injection was $81 \%$ in both the probiotic and control groups. This investigation was performed by sampling the yolk sac with a sterile cotton swab, streaking it on a BA and incubating aerobically overnight at $37^{\circ} \mathrm{C}$. None of the samples showed any bacterial growth. However, it was noticed that the probiotic solution used for dipping covered the eggs as a film after drying, thus leading to suffocation of the foetuses owing to the blocking of the pores in the eggshell.

\section{Body weight of chickens receiving Enterococcus faecium as a spray pre-hatch}

The mean body weight of chickens on days 1,4 and 7 were $45.47 \pm 3.54$ and $44.80 \pm 3.49 \mathrm{~g}(P=0.3014), 90.77 \pm 11.56$ and $92.10 \pm 10.06 \mathrm{~g}(P=0.6355)$ and $139.6 \pm 22.57$ and $144.0 \pm 18.60 \mathrm{~g}(P=0.6255)$ in the control and probiotictreated groups, respectively (Figure 4). However, no statistical difference in the body weight was observed between the groups within the three sampling days.

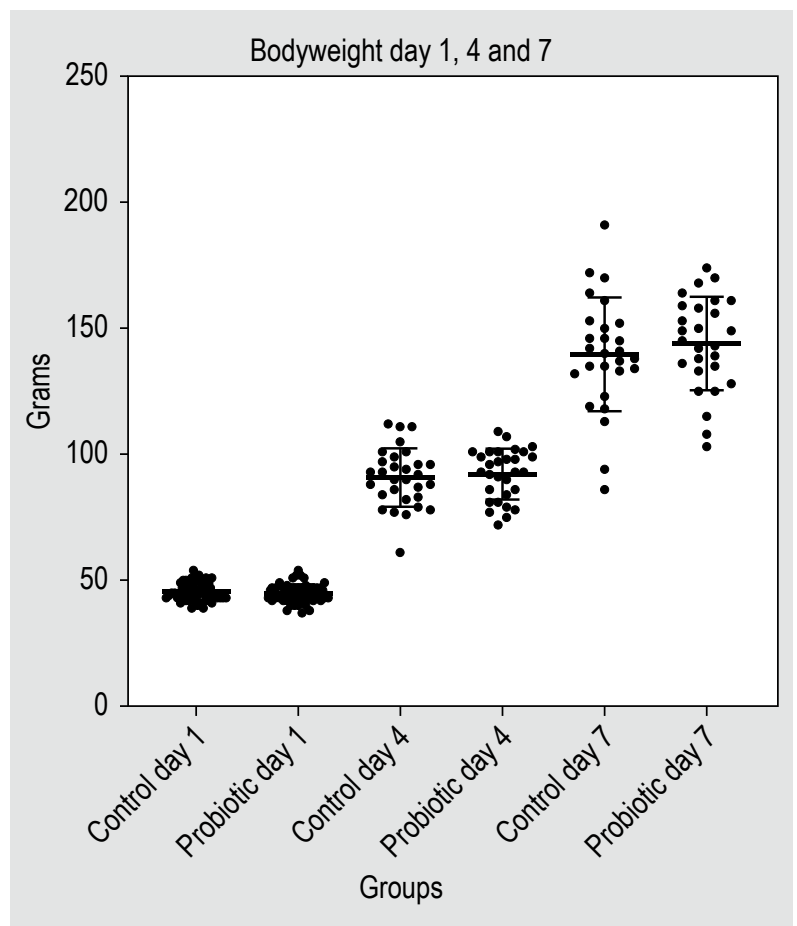

Figure 4. Mean bodyweight of the control $(n=30)$ and probiotictreated chicks ( $E$. faecium) $(n=30)$ on days 4 and 7 . No statistical difference was found between the control and probiotic-treated groups on days 1, 4 and 7 (Experiment 14) (Mean and standard deviation is shown).

\section{Discussion}

This study demonstrated that the probiotic E. faecium was transferred from the hen through the egg to the offspring by daily oral application of the probiotic to the hen $a d$ libitum (Objective 1). Before and right after hatch, 20 and $33 \%$ of the offspring were colonised, respectively. The higher percentage colonised at hatch indicated that the probiotic was spread horizontally in the hatcher during hatch (Objective 2). Such transmission has previously been described for Escherichia coli and Enterococcus faecalis, which is also proposed to be transmitted between chickens during hatch (Olsen et al., 2012; Poulsen et al., 2017). In terms of protection against bacterial pathogens in the first week of life, the ideal probiotic should be present, and colonising the gut of the chickens before the chicken is exposed to the pathogens is important. Thus, colonisation at the time of leaving the eggshell is proposed to be optimal timing. Moreover, an explanation for the relatively low colonisation rate $(33 \%)$ is proposed to be that the concentration of the probiotic was low on/in the eggs, which received the bacteria from the mother hen. However, neither the concentration on the eggshells nor that on the hen gut was investigated, thus needing further research. 
Furthermore, how long the colonisation time lasts after finalising the application of probiotic in drinking water was not assessed. Similarly, whether the colonisation of $33 \%$ of the chickens exerted a protective effect against the horizontal transmission of pathogens in the hatcher remains to be investigated. Therefore, the long-term effect of vertical transmission of probiotics on broiler performance needs further investigation as well. The probiotic was re-isolated from the salpinx of $50 \%$ of the investigated breeder hens, proposing that the bacteria ascended from the cloacal region into the oviduct/salpinx. The shell gland mucosa everts into the cloaca during ovipositioning and is thus exposed to the cloacal environment every time an egg is laid. In adult egg-laying birds, the major route of transmission in oviduct infections is proposed to be bacteria ascending from the gut or environment through the cloaca (Landman et al., 2013; Pors et al., 2014). However, the consequences/benefits of the presence of nonpathogenic bacteria in the oviduct remains unclarified. Additionally, it is unclear whether a distinct microbiome for the healthy egg-producing oviduct is present. Therefore, despite the small number of hens investigated, the presence of the probiotic in the oviduct (magnum and isthmus) suggests that a true vertical transmission into the egg occurs during egg formation. However, eggshell deposition of probiotic from the cloaca cannot be excluded either.

The ability of the probiotic strain to colonise the unhatched chickens when administered onto the eggshell on days 1618 of incubation varied from 0 to $9 \%$ (Objective 2). The lowest colonisation was found when probiotic $E$. faecium was administered in drops on the blunt end of the egg. However, the highest colonisation was found when the probiotic was administered as a spray (9\%). Furthermore, as described above, placing the drop at the blunt end did not result in colonisation of any chicken before hatch, whereas $7 \%$ were colonised when the drop was placed at the side of the egg. This result indicates that the E. faecium is transported by blood vessels present at the side of the egg but does not reach the blunt end where the air cell is present (Figure 1). The application by drops on the side of the eggs resulted in a lower colonisation rate after hatch than when spray was used to administer probiotic. This result accounts for the fact that the sampling occurred one day earlier and therefore, less time was allowed for horizontal transmission in the hatcher (Objective 3). Another explanation is that by drop application, only a limited area of the eggshell was exposed to the probiotic than the spray, where approximately $1 / 3$ to $1 / 2$ of the area of the shell is exposed. These hypotheses need verification by further investigations.

Additionally, when L. animalis was used as a probiotic, no pre-hatch colonisation occurred (Objective 4). Preliminary unpublished data showed that $L$. animalis survived for 4 days at $37^{\circ} \mathrm{C}$ under aerobic conditions at the shell of egg.
Therefore, the lack of colonisation is proposed to be owing to a lower ability to pass through the eggshell barriers and reach the foetus. In the newly hatched chickens sprayed with $L$. animalis, the bacteria could be re-isolated from $54 \%$ of the chickens (Objective 3 and 4).

Therefore, the results indicate that considerable colonisation occurs during hatch, resulting in a 2.8- to 11.5-fold increase in E. faecium and unmeasurable for L. animalis. At the time of hatch, chickens from the probiotic-treated eggs will be colonised when they start eating whatever soft and edible is present in the hatcher (e.g. shell membranes, meconium and egg content from exploded eggs) (Objective 3). Thus, the probiotic strain exerted protection by competitive exclusion, pathogen inhibition (De Oliveira et al., 2014; Varmuzova et al., 2016) and enhancement of gut integrity. However, during hatch, the chickens were colonised or exposed to pathogens, such as E. coli and E. faecalis together with the probiotic. The question of how important it is to be colonised by bacteria exerting a positive effect on gut health before exposure to pathogens requires more investigations.

The noninvasive application of probiotics did not affect the hatchability except for one experiment were eggs were dipped in a concentrated solution of E. faecium. The difference in hatchability among the experiments described in Tables 3, 4 and 5 most reflects that the eggs were provided by different hatcheries with different management procedures for storage, candling, sorting and so on of eggs.

The presence of the probiotic strain of E. faecium for 4 and 7 days after hatch at relatively high concentrations at both time points indicates that the bacteria survived. It shows that the bacteria colonised the intestinal tract of the newly hatched chicken for at least the first week of life (Objective 5). Comparison with in ovo injected birds was considered an application control for the spray application because in ovo injection makes it easier to control the concentration in all eggs (Objective 5). The caecal content of the probiotic was significantly higher in the caecal sacs of the chickens which received the probiotic as a spray on the eggshell, than in those which received the probiotic as in ovo injection. The cfu in the spray solution was 10 times higher than the cfu in the injected solution $\left(10^{8} \mathrm{vs} 10^{7} \mathrm{cfu} / \mathrm{egg}\right)$. Furthermore, the management of the two groups of treatments differed which makes it difficult to draw definite conclusions.

\section{Conclusions}

E. faecium was successfully recovered after vertical transmission from mother hen to offspring (Objective 1). The above described experiments showed that noninvasive application of an E. faecium probiotic was a successful method to colonise a proportion of the foetuses before hatch (Objectives 1 and 2). However, not all probiotic strains were equally successful (Objective 4 ). 
Furthermore, it can be concluded that the probiotics were horizontally transmitted during hatch and a high percentage of chickens were colonised at hatch. Moreover, the proportion of colonised chickens depended on the application method and the probiotic used (Objective 3).

Therefore, we propose that spraying the probiotics on fertilised eggs should be used as an 'easy-to-use' method for the noninvasive mass application of probiotics for both small and large hatcheries in the future (Objective 5).

Finally, alternative methods for the application of probiotics will be an advantage in broiler production as an alternative method to address the reduced hatchability when in ovo injection application of probiotics is used. It should extend to the use of probiotics to hatcheries which are not using the in ovo technology. Furthermore, this alternative, easy-to-use and relatively low-tech methods prove valuable for small scale/non-automated hatcheries and fancy breed breeders.

\section{Supplementary material}

Supplementary material can be found online at https://doi. org/10.3920/BM2020.0227.

Table S1. Summary of all application experiments.

Table S2. Preparation of mastermix for M74_F5/R5 (Enterococcus faecium, NCIMB11181) and LA51_F/R (Lactobacillus animalis, DSM33570).

Table S3. PCR programs for Enterococcus faecium (NCIMB11181) and Lactobacillus animalis (DSM33570).

\section{Acknowledgements}

We would like to thank Julie Svan Nielsson and Line Skjøt-Rasmussen for their scientific contributions during discussions of the study. Johanne Juul and Morten Dencker are thanked for their scientific contributions and sharing their knowledge about incubation and hatching. Erik Juncker Boll and Elke Brockman are thanked for designing and validating primers for the two probiotic strains. Mette and Peter Erdmann are thanked for housing broiler breeders for the vertical experiment. Dani Frost Clausen is thanked for the creative visualisation of the pre-hatch application methods.

\section{Conflicts of interest}

The work was co-funded by University of Copenhagen, DanHatch A/S, Chr. Hansen A/S and Green Development and Demonstration Programme, GUDP (Grant Number 34009-18-1360). IT, KA and LP are employed at UCPH, and DS is employed at Chr. Hansen A/S. One author is employed at Chr. Hansen A/S that produces the probiotic bacteria, which have been used in this study, resulting in a potential or perceived conflict of interest. However, the three independent academic partners (University of Copenhagen) explicitly approve that the work is at 'arm's length' from commercial interest. The first and senior author has led the process and is satisfied that the results are independent.

\section{References}

Aviagen, 2016. Parentstock, Ross 308, performance objectives. Aviagen, Huntsville, AL, USA. Available at: https://tinyurl.com/ypa43356

Aviagen. 2018. Parentstock, Ross 308, management handbook 2018. Aviagen, Huntsville, AL, USA. Available at: https://tinyurl. com/45xnuspw

De Oliveira, J.E., Van der Hoeven-Hangoor, E., Van de Linde, I.B., Montijn, R.C.,Van der Vossen, J.M.B.M., 2014. In ovo inoculation of chicken embryos with probiotic bacteria and its effect on posthatch Salmonella susceptibility. Poultry Science 93: 818-829.

Dominguez-Bello, M.G., Martin J.B., Ruth E.L., and Rob, K., 2011. Development of the human gastrointestinal microbiota and insights from high-throughput sequencing. Gastroenterology 140: 17131719. https://doi.org/10.1053/j.gastro.2011.02.011

Jørgensen, S.L., Poulsen, L.L., Thorndal, L, Ronaghinian, A.A., Bisgaard, M., Christensen, H., 2017. Characterization of Enterococcus faecalis isolated from the cloaca of 'fancy breeds' and confined chickens. Journal of Applied Microbiology 122: 1149-1158.

Kubasova, T., Kollarcikova, M., Crhanova, M., Karasova, D., Cejkova, D., Sebkova, A., Matiasovicova, J., Faldynova, M., Pokorna, A., Cizek, A. and Rychlik, I., 2019. Contact with adult hen affects development of caecal microbiota in newly hatched chicks. PLoS ONE 14: e0212446.

Landman, W.J.M., Heuvelink, A. and Van Eck, J.H.H. 2013. Reproduction of the Escherichia coli Peritonitis syndrome in laying hens. Avian Pathology 42: 157-162.

Oakley, B.B., Lillehoj, H.S., Kogut, M.H., Kim, W.K., Maurer, J.J., Pedroso, A., Lee, M.D., Collett, S.R., Johnson, T.J. and Cox, N.A., 2014. The chicken gastrointestinal microbiome. FEMS Microbiology Letters 360: 100-112.

Olsen, R.H., Christensen, H. and Bisgaard, M., 2012. Transmission and genetic diversity of Enterococcus faecalis during hatch of broiler chicks. Veterinary Microbiology 160: 214-221.

Patterson, J.A. and Burkholder, K.M., 2003. Application of prebiotics and probiotics in poultry production. Poultry Science 82: 627-631.

Peebles, E.D., 2018. In ovo applications in poultry: a review. Poultry Science 97: 2322-2338. https://doi.org/10.3382/ps/pey081

Pors, S.E., Olsen, R.H. and Christensen J.P. 2014. Variations in virulence of avian pathogenic Escherichia coli demonstrated by the use of a new in vivo infection model. Veterinary Microbiology 170: 368-374. https://doi.org/10.1016/j.vetmic.2014.02.043

Poulsen, L.L., Thøfner, I., Bisgaard, M., Christensen, J.P., Olsen, R.H. and Chistensen, H., 2017. Longitudinal study of transmission of Escherichia coli from broiler breeders to broilers. Veterinary Microbiology 207: 13-18. https://doi.org/10.1016/j. vetmic.2017.05.029 
Retes, P.L., Clemente A.H.S., Neves, D.G., Espósito, M., Makiyama, L., Alvarenga, R.R., Pereira, L.J. and Zangeronimo, M.G. 2018. In ovo feeding of carbohydrates for broilers - a systematic review. Journal of Animal Physiology and Animal Nutrition 102: 361-369.

Salminen, S. and Isolauri, E., 2006. Intestinal colonization, microbiota, and probiotics. Journal of Pediatrics 149: 115-120.

Skjøt-Rasmussen, L., Sandvang, D., Blanch, A., Nielsen J.M., Styrishave, T., Schnabl, J., Brockmann, E., Beck C.N. and Kiess, A.S. 2019. Post hatch recovery of a probiotic Enterococcus faecium strain in the yolk sac and intestinal tract of broiler chickens after in ovo injection. FEMS Microbiology Letters 366: fnz078.

Stanley, D., Geier, M.S., Hughes, R.J., Denman, S.E. and Moore, R.J., 2013. Highly variable microbiota development in the chicken gastrointestinal tract. PLoS ONE 8: e84290. https://doi.org/10.1371/ journal.pone.0084290
Stanley, D., Hughes, R.J. and Moore, R J., 2014. Microbiota of the chicken gastrointestinal tract: influence on health, productivity and disease. Applied Microbiology and Biotechnology 98: 4301-4310. https://doi.org/10.1007/s00253-014-5646-2

Timmerman, H.M., Veldman, A., Van den Elsen, E., Rombouts, F.M. and Beynen A.C., 2006. Mortality and growth performance of broilers given drinking water supplemented with chicken-specific probiotics. Poultry Science 85: 1383-1388.

Varmuzova, K., Kubasova, T., Davidova-Gerzova, L., Sisak, F., Havlickova, H., Sebkova, A., Faldynova, M. and Rychlik, I. 2016. Composition of gut microbiota influences resistance of newly hatched chickens to Salmonella enteritidis infection. Frontiers in Microbiology 7: 957.

Zulkifli, I., Abdullah, N., Azrin, N.M. and Ho, Y.W., 2000. Growth performance and immune response of two commercial broiler strains fed diets containing Lactobacillus cultures and oxytetracycline under heat stress conditions. British Poultry Science 41: 593-597. 
